\title{
CONTINUITY VERSUS NONEXISTENCE FOR A CLASS OF LINEAR STOCHASTIC CAUCHY PROBLEMS DRIVEN BY A BROWNIAN MOTION
}

\author{
JOHANNA DETTWEILER AND JAN VAN NEERVEN
}

\begin{abstract}
Let $A=d / d \theta$ denote the generator of the rotation group in the space $C(\Gamma)$, where $\Gamma$ denotes the unit circle. We show that the stochastic Cauchy problem

$$
d U(t)=A U(t)+f d b_{t}, \quad U(0)=0,
$$

where $b$ is a standard Brownian motion and $f \in C(\Gamma)$ is fixed, has a weak solution if and only if the stochastic convolution process $t \mapsto(f * b)_{t}$ has a continuous modification, and that in this situation the weak solution has a continuous modification. In combination with a recent result of Brzeźniak, Peszat and Zabczyk it follows that (1) fails to have a weak solution for all $f \in C(\Gamma)$ outside a set of the first category.
\end{abstract}

Over the past decades, a theory of stochastic differential equations in Hilbert spaces has been developed by many authors and is well documented in the monographs of Da Prato and Zabczyk [4, 5]. When trying to extend this theory to the Banach space setting, one immediately encounters the fundamental problem of setting up a theory of stochastic integration for Banach space-valued processes. For the class of Banach spaces with martingale type 2 (which includes, for example, the $L^{p}$-spaces for $2 \leqslant p<\infty$ ) this problem has been successfully addressed and a satisfactory theory of stochastic differential equations in these spaces is available. An overview with references to the literature is given in [1].

A theory of stochastic integration for functions with values in arbitrary Banach spaces which does not require any a priori geometric assumptions has been recently put forward in $[2,11]$, where it has been applied to linear stochastic differential equations driven by cylindrical Brownian motions. The main idea is to define the stochastic integral in a weak sense and to prove a version of the Itô isometry in which the $L^{2}$-norm of the stochastic integral

2000 Mathematics Subject Classification. Primary: 35R15 Secondary: 47D05, 60H15.

Key words and phrases. Stochastic linear Cauchy problems, nonexistence of weak solutions, continuous modifications, $C_{0}$-groups of linear operators.

The first named author was supported by the "Landesgraduiertenförderungsgesetz (LGFG) Baden-Württemberg" and the DAAD. The second named author gratefully acknowledges the support by a 'VIDI subsidie' in the 'Vernieuwingsimpuls' programme of the Netherlands Organization for Scientific Research (NWO) and the Research Training Network HPRN-CT-2002-00281. 
replaced by the radonifying norm of a certain integral operator canonically associated with the integrated function.

A problem that was left open in these works is that of the existence of a continuous modification of the solutions, even in the case where the equation is driven by a rank one Brownian motion. In the Hilbert space case, the existence of a continuous version of the solutions of linear equations driven by an arbitrary Brownian motion follows from the factorization method of Da Prato, Kwapień and Zabczyk; see [4, Chapter 5]. The present paper grew out an attempt to examine the situation in the Banach space setting for certain special cases where the semigroup generated by $A$ possesses minimal smoothing properties. To explain the main idea, let $C(\Gamma)$ denote the Banach space of continuous real-valued functions on the unit circle $\Gamma$. In a recent paper [3], Brzeźniak, Peszat, and Zabczyk showed that for 'most' functions $f \in C(\Gamma)$, the stochastic convolution with a standard real-valued Brownian motion $b=\left\{b_{t}\right\}_{t \geqslant 0}$,

$$
t \mapsto(f * b)_{t}=\int_{0}^{t} f(t-s \bmod 2 \pi) d b_{s},
$$

fails to have a modification with continuous trajectories. Indeed, the authors showed that the set of all $f \in C(\Gamma)$ for which such a modification exists is of the first category in $C(\Gamma)$. The main ingredient is a deep regularity result for random trigonometric series [7, Theorem 8.1]. This seems to suggest an approach towards a negative solution of the continuous modification problem for stochastic equations in $C(\Gamma)$. To see why, let $A=d / d \theta$ denote the generator of the rotation group $S=\{S(t)\}_{t \geqslant 0}$ in $C(\Gamma)$ and consider the problem

$$
\begin{aligned}
d U(t) & =A U(t) d t+f d b_{t}, \quad t \geqslant 0, \\
U(0) & =0,
\end{aligned}
$$

where $f \in C(\Gamma)$ is a given function. If this problem has a weak solution $\left\{U_{f}(t)\right\}_{t \geqslant 0}$ in $C(\Gamma)$ (in the sense of $[2,11]$ ), then for all $t \geqslant 0$ we have

$$
\left\langle U_{f}(t), \delta_{0}\right\rangle=\int_{0}^{t}\left\langle S(t-s) f, \delta_{0}\right\rangle d b_{s}=\int_{0}^{t} f(t-s \bmod 2 \pi) d b_{s}
$$

almost surely, where $\delta_{0}$ denotes the Dirac measure at 0. By the BrzeźniakPeszat-Zabczyk result, the right hand side fails to have a continuous modification for all functions $f$ outside a set of the first category in $C(\Gamma)$. Interestingly, however, it turns out that precisely for these $f$ the above problem fails to have a weak solution. This is the content of our main result, Theorem 1. This theorem shows that problem (2) actually provides an example of nonexistence and, at the same time, some evidence for a positive solution to the continuous modification problem.

Theorem 1. For a given function $f \in C(\Gamma)$, the problem (2) has a weak solution if and only if the convolution process $f * b$ has a modification with 
continuous trajectories, and in this situation the weak solution has a modification with continuous trajectories.

Let $(\Omega, \mathscr{F}, \mathbb{P})$ be a probability space with filtration $\left\{\mathscr{F}_{t}\right\}_{t \in[0, T]}$ and let $H$ be a real Hilbert space with inner product $[\cdot, \cdot]_{H}$. A cylindrical $H$-Wiener process on $(\Omega, \mathbb{P})$ indexed by the interval $[0, T]$ is a family $\mathbb{W}^{H}=\left\{W_{t}^{H}\right\}_{t \in[0, T]}$ of bounded linear operators from $H$ into $L^{2}(\Omega)$ with the following properties:

(1) For all $h \in H,\left\{W_{t}^{H} h\right\}_{t \in[0, T]}$ is an adapted Brownian motion;

(2) For all $s, t \in[0, T]$ and $g, h \in H$ we have

$$
\mathbb{E}\left(W_{s}^{H} g \cdot W_{t}^{H} h\right)=(s \wedge t)[g, h]_{H} .
$$

The noise term in (2) fits into this framework as follows:

Example 2. Suppose $E$ is a real Banach space and let $x \in E$ be a fixed nonzero element. Let $H$ denote the one-dimensional subspace spanned by $x$, endowed with the norm $\|c x\|_{H}:=|c|$. If $b=\left\{b_{t}\right\}_{t \in[0, T]}$ is a standard real-valued Brownian motion, then

$$
W_{t}^{H}(c x):=c b_{t}, \quad c \in \mathbb{R}
$$

defines a cylindrical $H$-Wiener process.

The 'only if' part of the theorem is a consequence of the following result, which gives some further support towards a positive solution to the continuous modification problem.

Proposition 3. Let $A$ be the generator of a $C_{0}$-group $\{S(t)\}_{t \geqslant 0}$ on a real Banach space E. Furthermore let $\left\{W_{t}^{H}\right\}_{t \geqslant 0}$ be a cylindrical $H$-Wiener process, where $H$ is a separable real Hilbert space, and let $B: H \rightarrow E$ be a bounded operator. If $\{U(t)\}_{t \geqslant 0}$ is a weak solution of the stochastic Cauchy problem

$$
\begin{aligned}
d U(t) & =A U(t) d t+B d W_{t}^{H}, \quad t \geqslant 0, \\
U(0) & =0,
\end{aligned}
$$

then $\{U(t)\}_{t \geqslant 0}$ has a modification with continuous trajectories.

The proof below is based upon the trivial observation that the group property implies that for all $0 \leqslant t \leqslant T$ we have

$$
\int_{0}^{t} S(t-s) B d W_{s}^{H}=S(t-T) \int_{0}^{t} S(T-s) B d W_{s}^{H} .
$$

This identity enables one to deduce properties of the stochastic convolution process from the corresponding properties of the stochastic integral process. The same idea was applied in [6] to the unitary dilations of Hilbert space contraction semigroups to obtain a maximal inequality for stochastic convolution of such semigroups with respect to a Hilbert space-valued Brownian motion.

Before turning to the proof of the proposition we need to give a meaning to the stochastic integrals in (4). We do this in two steps. First, the stochastic 
integral of a step function in $f \in L^{2}(0, T ; H)$ with respect to a cylindrical $H$-Wiener process $\mathbb{W}^{H}$ is defined in the obvious way, and by the Itô isometry

$$
\mathbb{E}\left|\int_{0}^{T} f(t) d W_{t}^{H}\right|^{2}=\int_{0}^{T}\|f(t)\|_{H}^{2} d t
$$

this definition extends to arbitrary functions $f \in L^{2}(0, T ; H)$. Second, an operator-valued function $\Phi:(0, T) \rightarrow \mathscr{L}(H, E)$ is said to be $H$-weakly $L^{2}$ if $\Phi^{*}(\cdot) x^{*} \in L^{2}(0, T ; H)$ for all $x^{*} \in E^{*}$, and stochastically integrable with respect to $\mathbb{W}^{H}$ if it is $H$-weakly $L^{2}$ and for every measurable set $A \subseteq(0, T)$ there exists a strongly measurable $E$-valued random variable $X_{A}$ such that for all $x^{*} \in E^{*}$ we have

$$
\left\langle X_{A}, x^{*}\right\rangle=\int_{0}^{T} 1_{A}(t) \Phi^{*}(t) x^{*} d W_{t}^{H}
$$

almost surely. The random variable $X_{A}$, if it exists, is determined uniquely almost everywhere and Gaussian. We call $X_{A}$ the stochastic integral of $\Phi$ over $A$, notation

$$
X_{A}=\int_{0}^{T} 1_{A}(t) \Phi(t) d W_{t}^{H} .
$$

For a systematic development of this integral we refer to [11], where it is shown that in the above definition it suffices to consider the set $A=(0, T)$. Note that if $\Phi$ is stochastically integrable on $(0, T)$, then $\Phi$ is stochastically on every subinterval $(0, t)$ and we have $\int_{0}^{t} \Phi(s) d W_{s}^{H}=\int_{0}^{T} 1_{(0, t)}(s) \Phi(s) d W_{s}^{H}$ almost surely.

Let us now assume that $H$ is separable and fix an orthonormal basis $\left(h_{n}\right)_{n=1}^{\infty}$ for $H$. Upon identifying $\mathscr{L}(\mathbb{R}, E)$ with $E$ in the canonical way, for each $n \geqslant 1$ the $E$-valued function $\Phi(\cdot) h_{n}$ is stochastically integrable with respect to the cylindrical $\mathbb{R}$-Wiener process (i.e., real Brownian motion) $\mathbb{W}_{(\cdot)}^{H} h_{n}$ and we have the 'coordinate expansion' [11, Theorem 4.2]

$$
X_{A}=\sum_{n=1}^{\infty} \int_{0}^{T} 1_{A}(t) \Phi(t) h_{n} d W_{t}^{H} h_{n}
$$

where the series converges unconditionally in $L^{2}(\Omega ; E)$.

Lemma 4. Let $H$ be separable and let $\Phi:(0, T) \rightarrow \mathscr{L}(H, E)$ be stochastically integrable with respect to the cylindrical $H$-Wiener process $\mathbb{W}^{H}$. Then the E-valued process

$$
Y_{t}:=\int_{0}^{t} \Phi(s) d W_{s}^{H}, \quad t \in[0, T],
$$

is a martingale which has a modification with continuous trajectories.

Proof. The martingale property is evident. To prove the existence of a continuous modification we fix an orthonormal basis $\left(f_{m}\right)_{m=1}^{\infty}$ in $L^{2}(0, T)$ 
and an orthonormal basis $\left(h_{n}\right)_{n=1}^{\infty}$ in $H$. For $N \geqslant 1$ we put

$$
Y^{(N)}:=\sum_{m, n=1}^{N} \int_{0}^{T} f_{m}(s) \Phi(s) h_{n} d s \int_{0}^{T} f_{m}(s) d W_{s}^{H} h_{n},
$$

where the first of these integrals exists as a Pettis integral; the Pettis integrability of the functions $t \mapsto f(t) \Phi(t) h$ for $f \in L^{2}(0, T)$ and $h \in H$ follows from the fact [11, Theorem 2.3] that the stochastic integrability of $t \mapsto \Phi(t) h$ implies the existence of a bounded operator $I_{\Phi, h}: L^{2}(0, T) \rightarrow E$ which satisfies

$$
\left\langle I_{\Phi, h} f, x^{*}\right\rangle=\int_{0}^{T} f(t)\left\langle\Phi(t) h, x^{*}\right\rangle d t, \quad f \in L^{2}(0, T), x^{*} \in E^{*} .
$$

For all $t \in[0, T]$,

$$
Y_{t}^{(N)}:=\mathbb{E}\left(Y^{(N)} \mid \mathscr{F}_{t}\right)=\sum_{m, n=1}^{N} \int_{0}^{T} f_{m}(s) \Phi(s) h_{n} d s \int_{0}^{t} f_{m}(s) d W_{s}^{H} h_{n} .
$$

In particular, for each $N \geqslant 1$ the process $t \mapsto Y_{t}^{(N)}$ has a version with continuous trajectories.

We claim that for each $t \in[0, T]$ we have $\lim _{N \rightarrow \infty} Y_{t}^{(N)}=Y_{t}$ in $L^{2}(\Omega ; E)$. For the reader's convenience we outline the proof, which follows an argument from [11]. By expanding $\left[\Phi^{*}(\cdot) x^{*}, h_{n}\right]_{H}$ with respect to the basis $\left(f_{m}\right)_{m=1}^{\infty}$ and using (5), for all $x^{*} \in E^{*}$ we have

$$
\left\langle Y_{t}, x^{*}\right\rangle=\sum_{n, m=1}^{\infty} \int_{0}^{T} f_{m}(s)\left\langle\Phi(s) h_{n}, x^{*}\right\rangle d s \int_{0}^{t} f_{m}(s) d W_{s}^{H} h_{n}
$$

with convergence in $L^{2}(\Omega)$; this convergence is unconditional since $\left(h_{\pi(n)}\right)_{n \geqslant 1}$ is an orthonormal basis for every permutation $\pi$ of the positive integers. The Itô-Nisio theorem [9, Theorem 2.1.1 (i) $\Leftrightarrow(\mathrm{v})$ and Theorem 2.2.1] now implies that

$$
Y_{t}=\sum_{n, m=1}^{\infty} \int_{0}^{T} f_{m}(s) \Phi(s) h_{n} d s \int_{0}^{t} f_{m}(s) d W_{s}^{H} h_{n}
$$

unconditionally in $L^{2}(\Omega ; E)$, and the claim follows.

The existence of a continuous modification of $Y$ now follows from a standard application of Doob's inequality.

Proof of Proposition 3: Fix $T \geqslant 0$. It is sufficient to show that the process $\{U(t)\}_{t \in[0, T]}$ has a continuous modification.

We know from $[2,11]$ that if a weak solution $\{U(t)\}_{t \geqslant 0}$ exists, it is unique, for every $t \geqslant 0$ the $\mathscr{L}(H, E)$-valued function $s \mapsto S(t-s) B$ is stochastically integrable on $(0, t)$, and $\{U(t)\}_{t \geqslant 0}$ is given by

$$
U(t)=\int_{0}^{t} S(t-s) B d W_{s}^{H}=S(t-T) \int_{0}^{t} S(T-s) B d W_{s}^{H}, \quad t \in[0, T] .
$$


By Lemma 4, the right hand side has a continuous modification on $[0, T]$.

Proof of Theorem 1: Using the construction of Example 2 we see that (2) is a special case of (3) by taking $H=\operatorname{span}\{x\}$ and $W_{t}^{H}(c x)=c b_{t}$, and defining $B_{f}: H \rightarrow C(\Gamma)$ by $B_{f}(c x):=c f$. By Proposition 3 and the observations at the beginning of the paper, (2) fails to have a weak solution whenever the convolution of $f$ with $b$ fails to have a continuous modification.

Let us now assume that, conversely, the convolution process $f * b$ has a continuous modification. Then the convolution process $t \mapsto(f * \tilde{b})_{t}$ has a continuous modification as well, where $\tilde{b}_{t}:=b_{2 \pi+t}-b_{2 \pi}$. Indeed, this may be deduced from [8, Lemma 3.24] or from a general comparison result for Gaussian processes [10, Theorem 12.16]. Now define, for $\theta \in \Gamma$,

(6) $X_{f}(\theta):=\int_{0}^{2 \pi+\theta} f(2 \pi+\theta-s \bmod 2 \pi) d b_{s}-\int_{0}^{\theta} f(\theta-s \bmod 2 \pi) d \tilde{b}_{s}$,

where on the right hand side we take the continuous modifications, and notice that

$$
X_{f}(\theta)=\int_{0}^{2 \pi} f(\theta-s \bmod 2 \pi) d b_{s}
$$

almost surely. Hence by the Pettis measurability theorem and the stochastic Fubini theorem, (6) defines a centred $C(\Gamma)$-valued Gaussian random variable $X_{f}$, and for any finite Borel measure $\mu \in(C(\Gamma))^{*}$ the variance of $\left\langle X_{f}, \mu\right\rangle$ is given by

$$
\begin{aligned}
\mathbb{E}\left\langle X_{f}, \mu\right\rangle^{2} & =\mathbb{E}\left(\int_{0}^{2 \pi} \int_{0}^{2 \pi} f(\theta-s \bmod 2 \pi) d b_{s} d \mu(\theta)\right)^{2} \\
& =\mathbb{E}\left(\int_{0}^{2 \pi} \int_{0}^{2 \pi} f(\theta-s \bmod 2 \pi) d \mu(\theta) d b_{s}\right)^{2} \\
& =\int_{0}^{2 \pi}\left(\int_{0}^{2 \pi} f(\theta-s \bmod 2 \pi) d \mu(\theta)\right)^{2} d s \\
& =\left\langle Q_{f} \mu, \mu\right\rangle .
\end{aligned}
$$

Here, the operator $Q_{f} \in \mathscr{L}\left(C(\Gamma)^{*}, C(\Gamma)\right)$ is defined by

$$
Q_{f} \mu:=\int_{0}^{2 \pi} S(t) B_{f} B_{f}^{*} S^{*}(t) \mu d t .
$$

The existence of a global weak solution $U_{f}$ now follows from [11, Corollary 7.2], cf. also [2, Theorem 5.3]

Remark 5. It is not hard to see that the solution $U_{f}$ is given by $U_{f}(t, \theta)=$ $\int_{0}^{t} f(t+\theta-s \bmod 2 \pi) d b_{s}$ almost surely.

Remark 6. Also in the space $L^{p}(\Gamma)$ with $1 \leqslant p<2$, the problem (2) fails to have a weak solution for 'most' functions $f \in L^{p}(\Gamma)$. More precisely, as a consequence of the Kahane-Khinchine inequalities it was shown in [11] that in this situation a weak solution exists if and only if $f \in L^{2}(\Gamma)$. 
Remark 7. We have seen in Proposition 3 that the existence of a weak solution $U$ to problem (3) implies the existence of a continuous modification of $U$ whenever $A$ is the generator of a $C_{0}$-group on $E$. Another situation where this is known to happen is the case where $A$ generates an analytic $C_{0}$-semigroup on $E$; see [2, Proposition 4.3, Theorem 6.1].

Acknowledgment - This work was carried out during a half-year stay of the first named author at the Technical University of Delft. She would like to thank her colleagues at the Department of Applied Mathematical Analysis for their kind hospitality. The authors thank the anonymous referee for suggesting some improvements in the presentation.

\section{REFERENCES}

[1] Z. BrzeźnIAK, Some remarks on stochastic integration in 2-smooth Banach spaces, in: "Probabilistic Methods in Fluids" (Eds. I.M. Davies, A. Truman et. al.), World Scientific, New Jersey, 2003, 48-69.

[2] Z. Brzeźniak And J.M.A.M. van NeErven, Stochastic convolution in separable Banach spaces and the stochastic linear Cauchy problem, Studia Math. 143 (2000), 43-74.

[3] Z. Brzeźniak, Sz. Peszat, J. Zabczyk, Continuity of stochastic convolutions, Czechoslovak Math. J. 51 (2001), 679-684.

[4] G. Da Prato And J. Zabczyk, "Stochastic Equations in Infinite Dimensions", Encyclopedia of Mathematics and its Applications, Cambridge University Press, Cambridge, 1992.

[5] G. Da Prato and J. Zabczyk, "Ergodicity for Infinit-Dimensional Systems", London Math. Soc. Lect. Note Series, Vol. 229, Cambridge University Press, Cambridge, 1996.

[6] E. Hausenblas And J. Seidler, A note on maximal inequality for stochastic convolutions, Czechoslovak Math. J. 51 (2001), 785-790.

[7] J.-P. Kahane, "Some Random Series of Functions", Second edition, Cambridge Studies in Advanced Mathematics, Vol. 5, Cambridge University Press, Cambridge, 1985.

[8] O. Kallenberg, "Foundations of Modern Probability", Second edition, Probability and its Applications, Springer-Verlag, New York, 2002.

[9] S. Kwapień and W.A. Woyczyński, "Random Series and Stochastic Integrals: Single and Multiple", Probability and its Applications, Birkhäuser Verlag, Boston, 1992.

[10] M. Ledoux and M. Talagrand, "Probability in Banach Spaces", Ergebnisse d. Math. u. ihre Grenzgebiete, Vol. 23, Springer-Verlag, 1991.

[11] J.M.A.M. VAN NEERVEn AND L. WEIS, Stochastic integration of functions with values in a Banach space, to appear in Studia Math.

Mathematisches Institut I, Technische Universität Karlsruhe, D-76128

Karlsruhe, Germany

E-mail address: Johanna.Dettweiler@mathematik.uni-karlsruhe.de

Department of Applied Mathematical Analysis, Technical University of Delft, P.O. Box 5031, 2600 GA Delft, The Netherlands

E-mail address: J.vanNeerven@math.tudelft.nl 